Supporting Information

\title{
One-Step Fabrication of Stretchable Copper Nanowire Conductors by a Fast Photonic Sintering Technique and Its Application in Wearable Devices
}

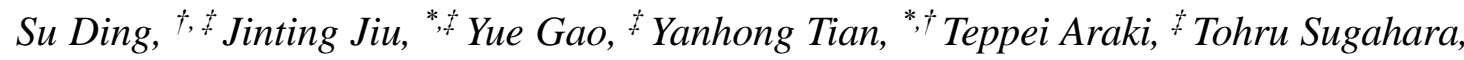

${ }^{\star}$ Shijo Nagao, ${ }^{*}$ Masaya Nogi ${ }^{\ddagger}$ Hirotaka Koga, ${ }^{\ddagger}$ Katsuaki Suganuma ${ }^{\ddagger}$ and Hiroshi Uchida $^{\S}$

${ }^{\dagger}$ State Key Laboratory of Advanced Welding and Joining, Harbin Institute of Technology, Harbin, 150001, China

\$ The Institute of Scientific and Industrial Research (ISIR), Osaka University, Osaka, 565-0871, Japan

$\S$ Institute for Polymers and Chemicals Business Development Center, Showa Denko K.K., Ichihara, Chiba 290-0067, Japan

Corresponding authors: tianyh@hit.edu.cn; jiu@eco.sanken.osaka-u.ac.jp 

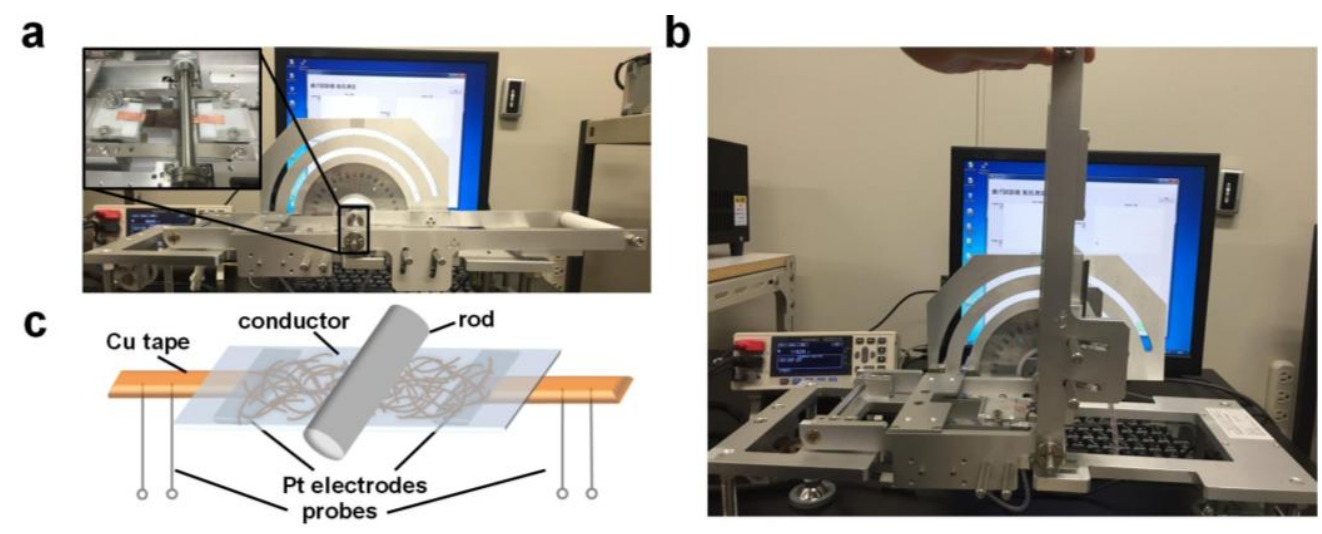

Figure S1. Photo of the home-made bending machine when the angle was set as (a) $0^{\circ}$ and (b) $90^{\circ}$, respectively. Inset in (a) shows the layout of CuNW conductor on the bending machine. (c) The schematic illustration of the bending experiment. CuNW conductors were put under the rod (diameter of $20 \mathrm{~mm}$ ) with front side downward. The ends of the conductor with sputtered $\mathrm{Pt}$ electrodes were connected to four probes through $\mathrm{Cu}$ tapes. The resistance was recorded during the cyclical bending process. 


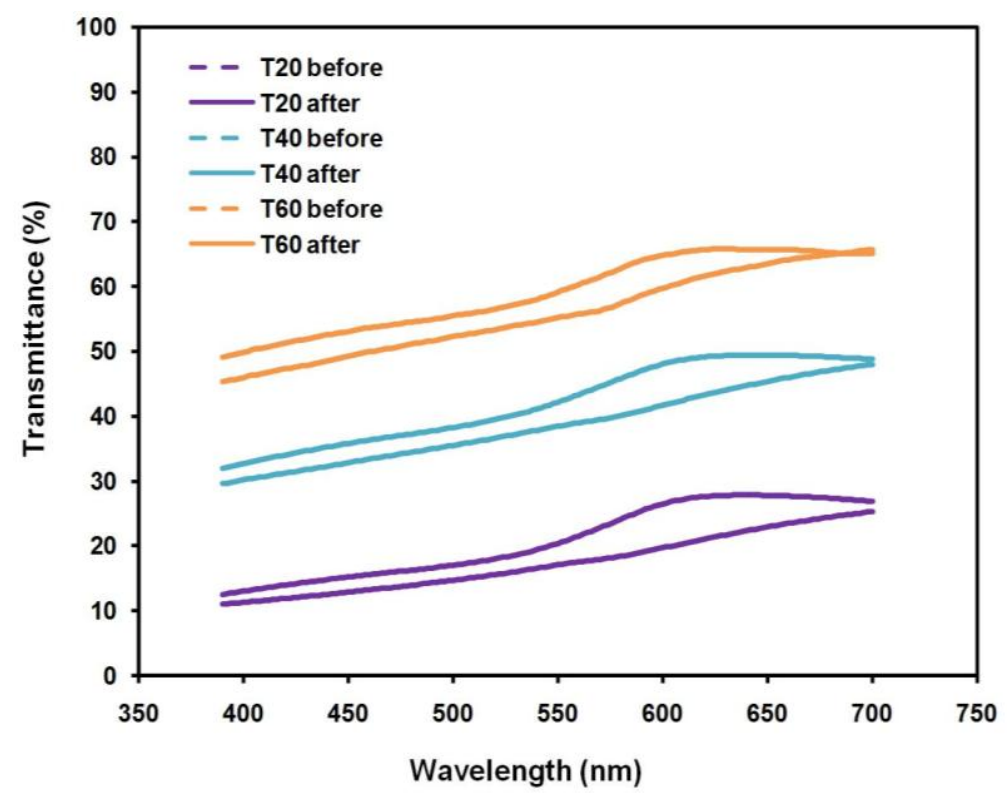

Figure S2. Transmittance spectrum of CuNW/PU conductors with various transmittance before and after the photonic sintering. 


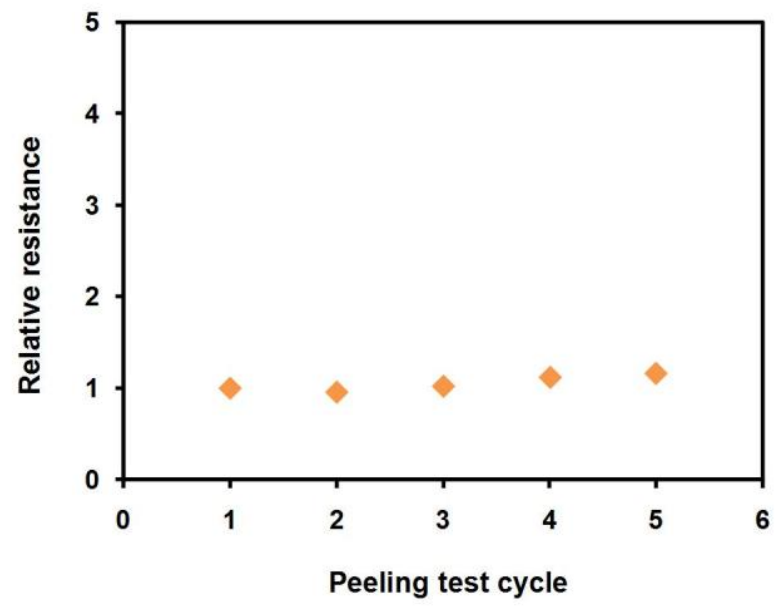

Figure S3. Variations in sheet resistance of CuNW/PU conductors as a function of peeling cycles. 

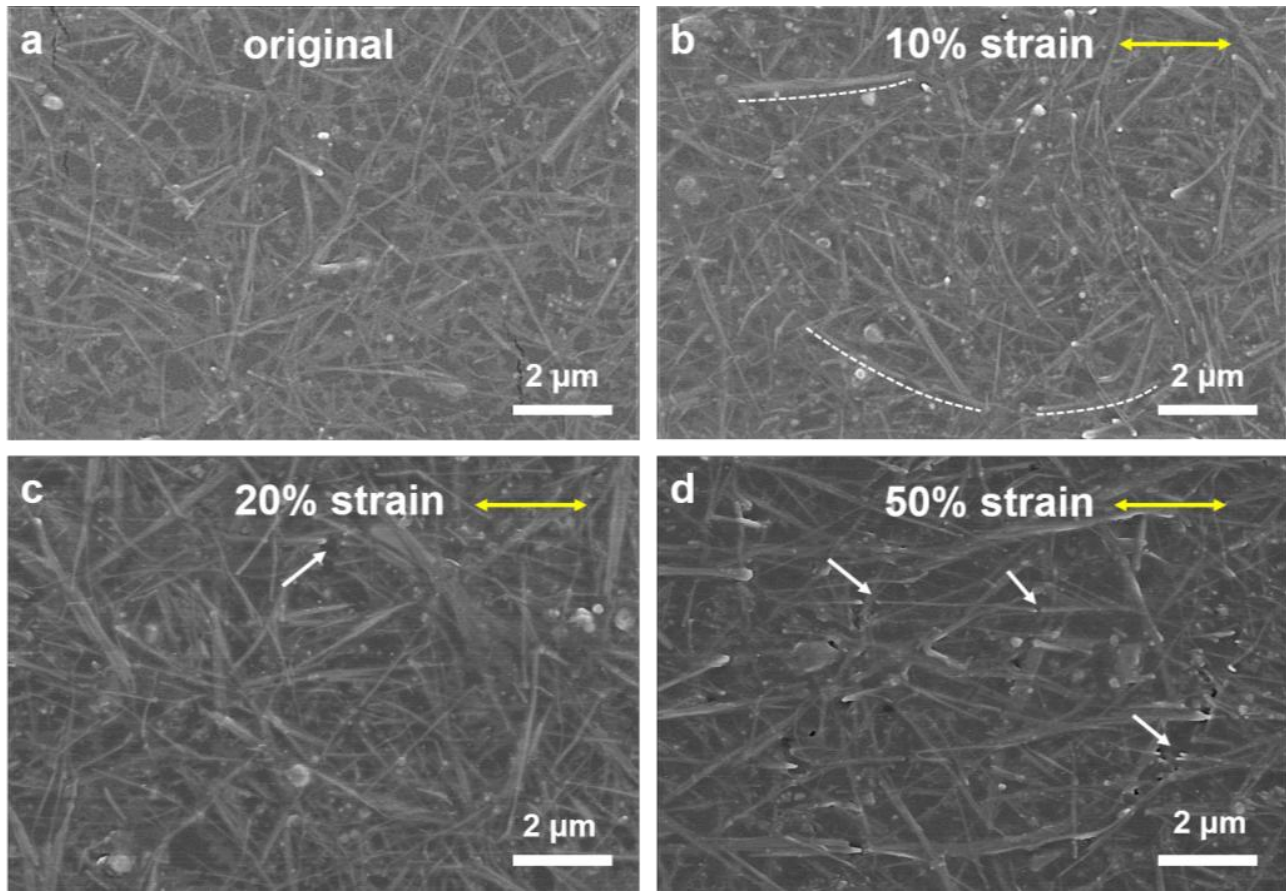

Figure S4. SEM images of CuNW network on PU substrate under various strains. (a) without strain, (b) $10 \%$ strain, (c) $20 \%$ strain, (d) $50 \%$ strain. The yellow double headed arrows show the direction of stretching strain. 


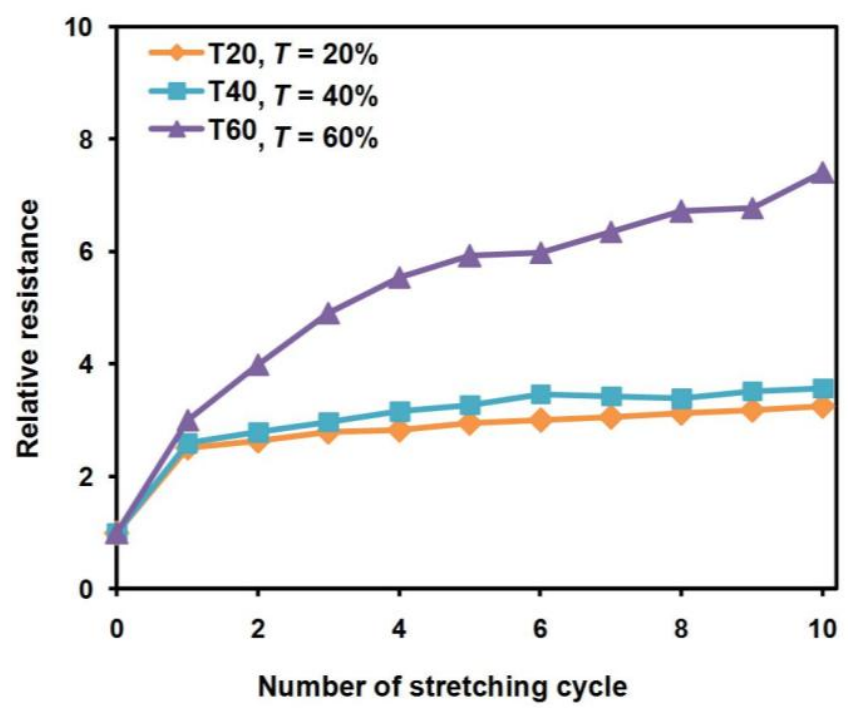

Figure S5. Relative resistance variation for T20, T40 and T60 samples under stretching statue at $10 \%$ strain for 10 cycles. 


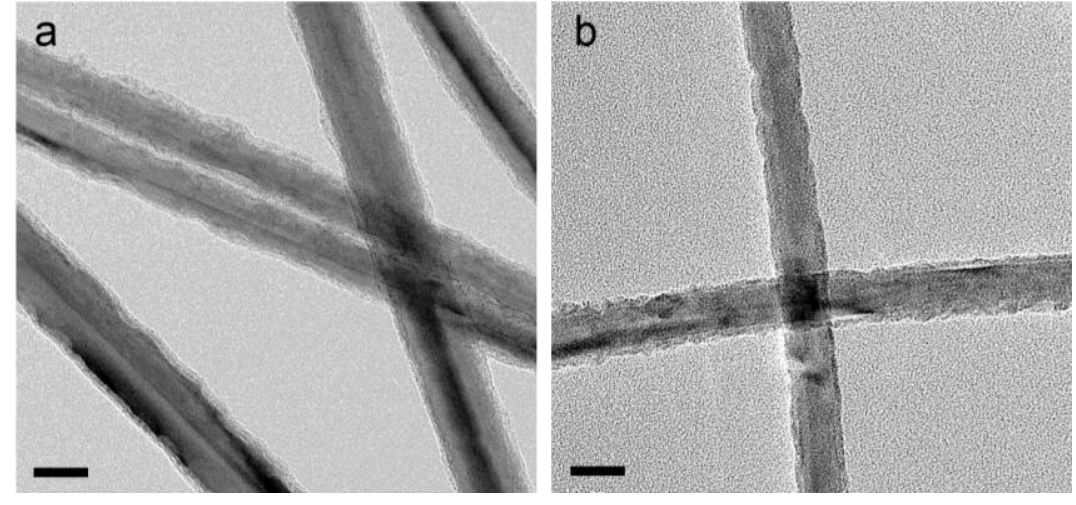

Figure S6. TEM images of fresh prepared CuNWs before (a) and after (b) photonic sintering process. The scale bar is $20 \mathrm{~nm}$. 


\section{Supporting movies}

Video S1. Movement of fingers with order of middle-index-middle-index to output the electrical signals of "c" (Morse code was dash-dot-dash-dot).

Video S2. Movement of fingers with order of index-index-middle to output the electrical signals of Morse code "u" (Morse code was dot-dot-dash). 